\title{
ESTATINAS HIPOLIPÊMICAS E NOVAS TENDÊNCIAS TERAPÊUTICAS
}

\section{Vanessa Leiria Campo e Ivone Carvalho*}

Departamento de Ciências Farmacêuticas, Faculdade de Ciências Farmacêuticas de Ribeirão Preto, Universidade de São Paulo, Av. do Café, s/n, 14040-903 Ribeirão Preto - SP, Brasil

Recebido em 12/9/05; aceito em 24/3/06; publicado na web em 30/8/06

HYPOLIPEMIC STATINS AND NEW THERAPEUTCAL TRENDS. Statins are the most used drugs for the treatment of hyperlipidemia in primary and secondary prevention, with the aim of decreasing the levels of plasmatic cholesterol- lipoproteins. Owing to their structural similarity to the substrate HMG-CoA (3-hydroxy-3-methylglutaryl-CoA), they inhibit the HMG-CoA reductase enzyme, disrupting the cholesterol biosynthesis. Currently, six therapeutic statins are available: lovastatin (Mevacor) and pravastatin (Pravachol), which are natural, sinvastatin (Zocor), a semi-synthetic derivative, and the totally synthetic statins, fluvastatin (Lescol), atorvastatin (Lipitor) and rosuvastatin (Crestor). Recent investigations have showed other important effects of statins, such as antineoplastic action and improvement in endothelial function.

Keywords: statins; cholesterol; HMG-CoA reductase.

\section{INTRODUÇÃO}

O número de mortes causadas por doenças cardiovasculares e, em especial, por doença cardíaca coronariana, tem declinado significativamente, mas as doenças cardiovasculares continuam sendo as responsáveis pelo alto índice de mortalidade e morbidade em homens com idade superior a 45 anos e mulheres acima de 65 anos. O número de mortes por doenças cardiovasculares no mundo todo, no ano de 2002, foi de 16,7 milhões de pessoas, sendo que as doenças cardíacas coronárias foram responsáveis por 7,2 milhões das mortes verificadas ${ }^{1}$. Nos Estados Unidos, cerca de 215.000 pessoas morrem a cada ano de doença cardiovascular, com 650.000 novos casos de infarto do miocárdio ${ }^{2,3}$. No Brasil, de acordo com o anuário estatístico de 2001, 257.179 pessoas morreram no ano de 1999 devido a doenças do aparelho circulatório, representando $32,3 \%$ dos óbitos registrados. Deste valor, 76.639 mortes foram causadas por doenças isquêmicas do coração, 83.475 por doença cerebrovascular e 97.065 devido às demais doenças do aparelho circulatório ${ }^{4}$. Em 2002, o número de mortes no Brasil, envolvendo doenças cardíacas, foi de $139.601^{1}$.

Diversos estudos epidemiológicos envolvendo pacientes hiperlipêmicos com excesso de lipídios no sangue têm mostrado uma relação direta entre o risco de desenvolvimento de doença arterial coronariana (DAC) ("Coronary Heart Disease", CHD) e lesões ateroscleróticas ${ }^{5}$, devido aos altos níveis de lipídios sanguíneos, geralmente na forma de lipoproteínas. A hiperlipidemia ou hiperlipemia pode ser causada por distúrbio genético, influências ambientais ou por uma combinação destes fatores.

As estatinas são os fármacos mais usados para tratamento das hiperlipidemias em prevenção primária e secundária, com o propósito de diminuir os níveis de lipoproteínas plasmáticas ricas em colesterol e reduzir os riscos de DAC. Estes efeitos são resultantes da atividade inibidora das estatinas sobre a enzima HMG-CoA redutase (hidroximetilglutaril-CoA redutase), com a propriedade de bloquear a conversão do substrato HMG-CoA em ácido mevalônico, inibindo os primeiros passos da biossíntese de colesterol. Estas substâncias, capazes de mimetizar o substrato natural, podem ser divi-

*e-mail: carronal@usp.br didas em naturais e sintéticas e diferem fundamentalmente em termos de potência, perfil farmacocinético, interação farmacológica e efeito indesejado relacionado à miotoxicidade.

Mevastatina (ou compactina) foi o primeiro inibidor de HMGCoA redutase descoberto em 1976, originalmente isolado como produto metabólico de culturas de Penicillium citrinium ${ }^{6}$, sendo sua afinidade pelo sítio enzimático cerca de 10.000 vezes superior ao substrato HMG-CoA. Por outro lado, lovastatina (ou mevinolina) foi isolada posteriormente de culturas de Aspergillus terreus e Monascus ruber com estrutura semelhante à mevastatina (grupo 6'-metílico adicional), mas com potência superior. Em 1987 lovastatina foi aprovada pelo FDA para uso terapêutico, enquanto mevastatina foi abandonada devido a problemas na morfologia intestinal de cachorros e toxicidade hepatocelular verificada em ratos.

A introdução de novos derivados sintéticos da classe das estatinas ocorreu em 1996 e 1997, com atorvastatina (Lipitor) e cerivastatina (Baycon), respectivamente. No entanto, em agosto de 2001, a Bayer retirou cerivastatina do mercado devido à incidência de mais de 100 casos de rabdomiólise fatal, relacionados a dano severo da musculatura esquelética com alteração das células musculares, liberação de mioglobina e, freqüentemente, acompanhado de problemas renais ${ }^{7}$. A maioria dos casos foi observada em tratamentos com doses excessivas de cerivastatina ou em doses normais associadas ao uso de genfibrosil, fármaco da classe dos fibratos empregados no tratamento das hipertrigliceridemias ${ }^{8}$.

Investigações recentes têm mostrado diversos efeitos importantes de cerivastatina e outras estatinas, como ação antineoplásica ${ }^{9}$, inibição da reabsorção óssea ${ }^{10}$, aumento da disponibilidade de óxido nítrico endotelial ${ }^{11}$, relaxamento do músculo sanguíneo ${ }^{12}$, atenuação da hipertrofia do miócito cardíaco induzida pelo endotélio ${ }^{13}$, inibição da proliferação de células endoteliais induzidas por fatores angiogênicos ${ }^{14}$, diminuição dos níveis de citocina pró-inflamatória IL-6, liberada pelo tecido adiposo $0^{6,15}$.

Em 2002, atorvastatina foi o fármaco mais vendido no mundo, rendendo para o fabricante Pfizer cerca de US\$ 8 bilhões ${ }^{16}$. Rosuvastatina (AstraZeneca), aprovada em agosto de 2003 pelo FDA, deverá concorrer por este mercado, enquanto pitavastatina, nova estatina sintética em estudos pela empresa japonesa Kowa Company, apresentou grande eficácia em reduzir os níveis de colesterol, com 
doses diárias de 1 a 4 mg e ação similar ou superior à atorvastatina $^{17,18}$.

No momento, seis estatinas são empregadas clinicamente: lovastatina (Mevacor), pravastatina (Pravachol), sinvastatina (Zocor), derivado semi-sintético, e fluvastatina (Lescol), primeiro agente totalmente sintético, derivado de mevalonolactona produzido na forma racêmica ${ }^{19}$. A nova geração de estatinas sintéticas, enantiomericamente puras, é representada por atorvastatina (Lipitor) e rosuvastatina (Crestor), (Figura 1).

Naturais

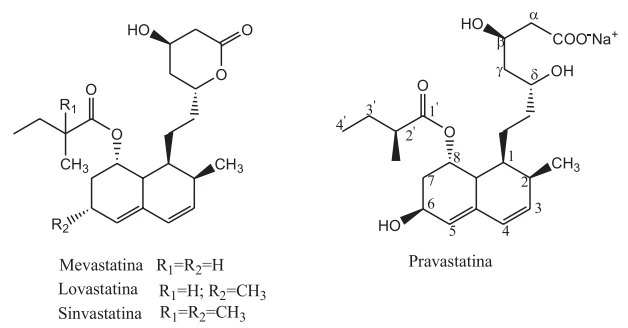

Sintéticos
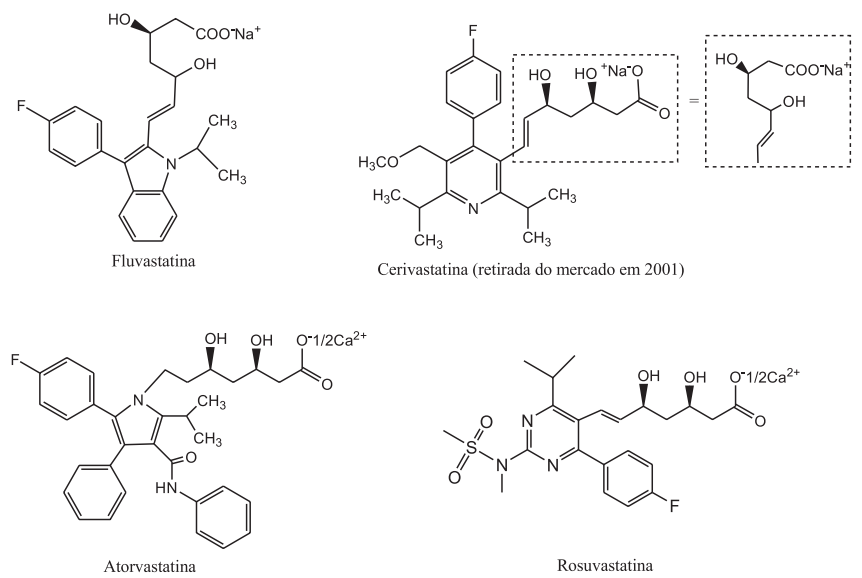

Figura 1. Estrutura química das estatinas inibidoras da enzima HMG-CoA redutase

Os efeitos de diminuição de LDL-colesterol em pacientes hipercolesterolêmicos parecem ser semelhantes entre as estatinas clássicas, ou seja, cerca de 25 a $45 \%$, dependendo da dose. No entanto, lovastatina e sinvastatina parecem mais vantajosas em relação à pravastatina porque produzem maior nível de HDL-colesterol e maior diminuição de triglicerídeos séricos, triglicerídeos VLDL e colesterol VLDL.

Atualmente, diversos dados da literatura mostram que as estatinas não só promovem diminuição de LDL-colesterol e aumento de HDL-colesterol, como também conduzem à diminuição dos níveis de triglicerídeos ${ }^{7-10}$. De forma geral, as estatinas causam redução dos níveis de triglicerídeos, mas este efeito parece ser observado apenas em pacientes hipertrigliceridêmicos. Os valores praticamente constantes obtidos da relação triglicerídeos/LDL-colesterol, nestes estudos, mostram que quanto maior a atividade de estatina para diminuir o LDL-colesterol, maior será o efeito na diminuição dos níveis de triglicerídeos ${ }^{12}$.

Lovastatina, pravastatina e, mais recentemente, sinvastatina apresentaram atividade antiaterogênica, relacionada à melhora da disfunção endotelial e vasomotora, diminuição da formação de trombos plaquetários e modulação na atividade fibrinolítica em pacientes hipercolesterolêmicos. Estes efeitos contribuem para re- dução de quadros de isquemia do miocárdio e incidência de infarto, com conseqüente diminuição do índice de mortalidade total causado por doenças cardiovasculares ${ }^{2}$.

As estatinas são bem toleradas com boa margem de segurança durante a utilização prolongada. Os efeitos colaterais não são freqüentes, mas um dos mais sérios está relacionado à ação hepatotóxica, com possível aumento das enzimas aspartato e alanina transaminases. Miopatias, com evolução a rabdomiólises e insuficiência renal, são raras, mas são efeitos graves associados ao uso de estatinas. Parece que o uso concomitante de estatinas com eritromicina, ciclosporina, niacina e fibratos aumenta o risco de miopatias em $10-30 \%$ dos pacientes.

\section{COLESTEROL E HMG-CoA REDUTASE}

Colesterol é um componente esteróide da membrana celular e um precursor importante dos hormônios andrógenos, estrogênios, progesterona e adrenocorticóides. A primeira etapa da biossíntese de colesterol no organismo é realizada pela associação de três moléculas de acetil-Coenzima A (acetil-CoA) para formação de 3hidróxi-3-metilglutaril-CoA (HMG-CoA). A conversão seguinte de HMG-CoA em ácido mevalônico é catalisada pela enzima HMGCoA redutase e representa a etapa mais importante de controle da biossíntese de colesterol no organismo (Figura 2).
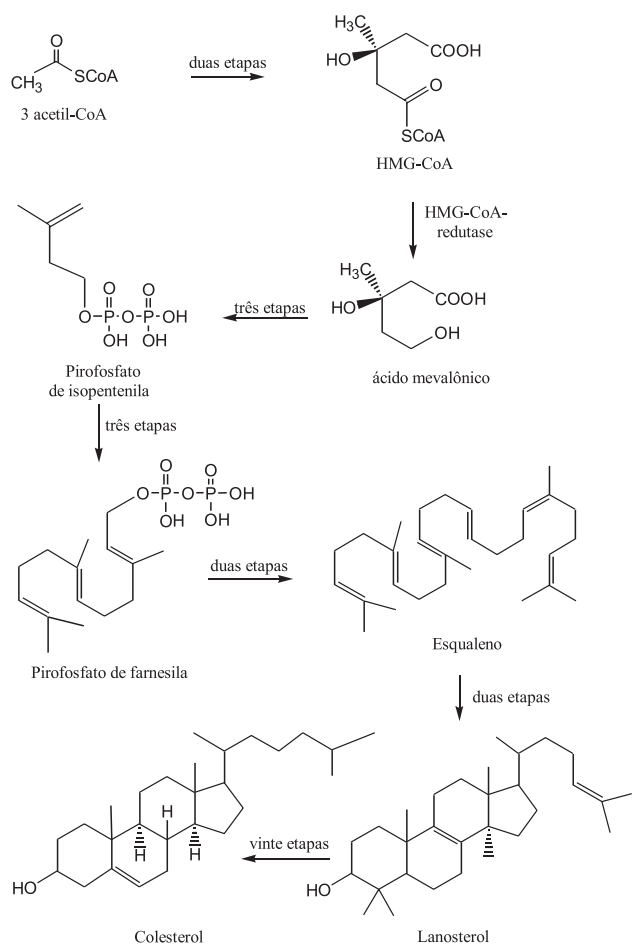

Figura 2. Biossíntese de colesterol a partir de acetil $\mathrm{CoA}$

A condensação de três moléculas de pirofosfato de isopentenila $\left(\mathrm{C}_{5}\right)$, gerado a partir de ácido mevalônico $\left(\mathrm{C}_{6}\right)$, fornece o intermediário pirofosfato de farnesila contendo estrutura de 15 átomos de carbono. A reação entre duas moléculas do intermediário isoprenóide $\mathrm{C}_{15}$ gera o derivado esqualeno $\mathrm{C}_{30}$. Esqualeno sofre ciclização devido à reação inicial de epoxidação, movimentação de elétrons e migração de dois grupos metilas. A conversão de lanosterol em colesterol ocorre em vinte etapas, a partir da remoção de três grupos metila, redução da dupla ligação da cadeia lateral e movimentação de dupla ligação no interior do anel de lanosterol ${ }^{20}$. 
Colesterol é metabolizado por dois caminhos envolvendo as enzimas desmolase e $7 \alpha$-hidroxilase. Pregnenolona é o produto da reação de clivagem oxidativa de colesterol pela desmolase, que atua como intermediário comum na biossíntese de todos os outros esteróides endógenos. Colesterol pode também ser convertido a ácidos e sais biliares pela atuação da enzima $7 \alpha$-hidroxilase, representando o mecanismo mais importante do catabolismo de colesterol. Ácido cólico e seus derivados constituem 99\% destes sais biliares e podem ser conjugados a moléculas de glicina ou taurina. Estes compostos são sintetizados no fígado, armazenados na bexiga e liberados no intestino delgado para emulsificar lipídios da dieta e vitaminas lipossolúveis, promovendo a absorção destes compostos através da mucosa intestinal. Sais biliares são predominantemente reabsorvidos pela circulação entero-hepática e retornam ao fígado para exercer um controle de "feedback" negativo sobre a enzima $7 \alpha$-hidroxilase e regular o metabolismo de colesterol.

A diminuição de colesterol plasmático é acompanhada pela redução dos níveis de LDL. Apesar da importância deste mecanismo no efeito hipocolesterolêmico, as estatinas também induzem o aumento de receptores LDL, onde a lipoproteína LDL se fixa no tecido hepático, ocorrendo depuração de partículas com apo B-100, por endocitose. $\mathrm{O}$ aumento de atividade dos receptores de LDL hepático é um dos fatores mais importantes para diminuição dos níveis de LDL plasmático. Por esta razão os inibidores de HMG-CoA redutase possuem dupla ação, diminuem a biossíntese de colesterol e causam aumento do número de receptores LDL hepático, aumentando a remoção de IDL e LDL circulantes.

\section{Mecanismo de ação}

Como ilustrado na Figura 3, o substrato HMG-CoA é formado a partir da combinação de acetil-CoA com acetoacetil-CoA e, subseqüentemente, sofre duas reações de redução, envolvendo a transferência de hidreto, fornecidas por duas moléculas de nicotinamida adenina difosfato (NADPH), catalisada pela enzima HMG-CoA redutase.
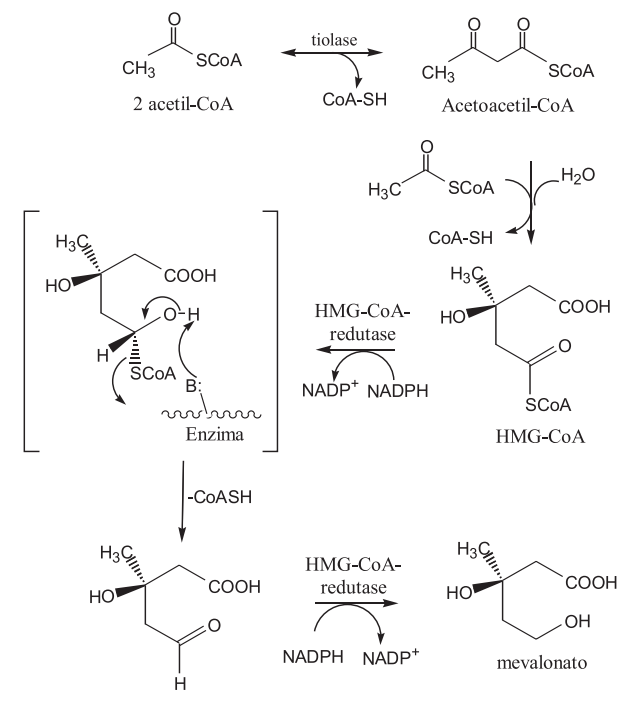

Figura 3. Biossíntese de mevalonato a partir de acetil-CoA, primeira etapa na biossíntese de novo de colesterol

A primeira reação de redução, na presença de NADPH, envolve a formação do intermediário hemi-acetal de mevaldil-CoA, que se decompõe a mevaldeído e CoASH, posteriormente protonado por grupo doador de hidrogênio no sítio catalítico (His866). Na terceira etapa, $\mathrm{NADP}^{+}$gerado anteriormente é substituído por outra molécula de NADPH para promover a segunda reação de redução, ou seja, conversão de mevaldeído a mevalonato. O mecanismo de ação molecular de redução do substrato, na presença de NADPH, é mostrado de forma detalhada na Figura 4, dando destaque aos aminoácidos do sítio catalítico envolvidos na reação.
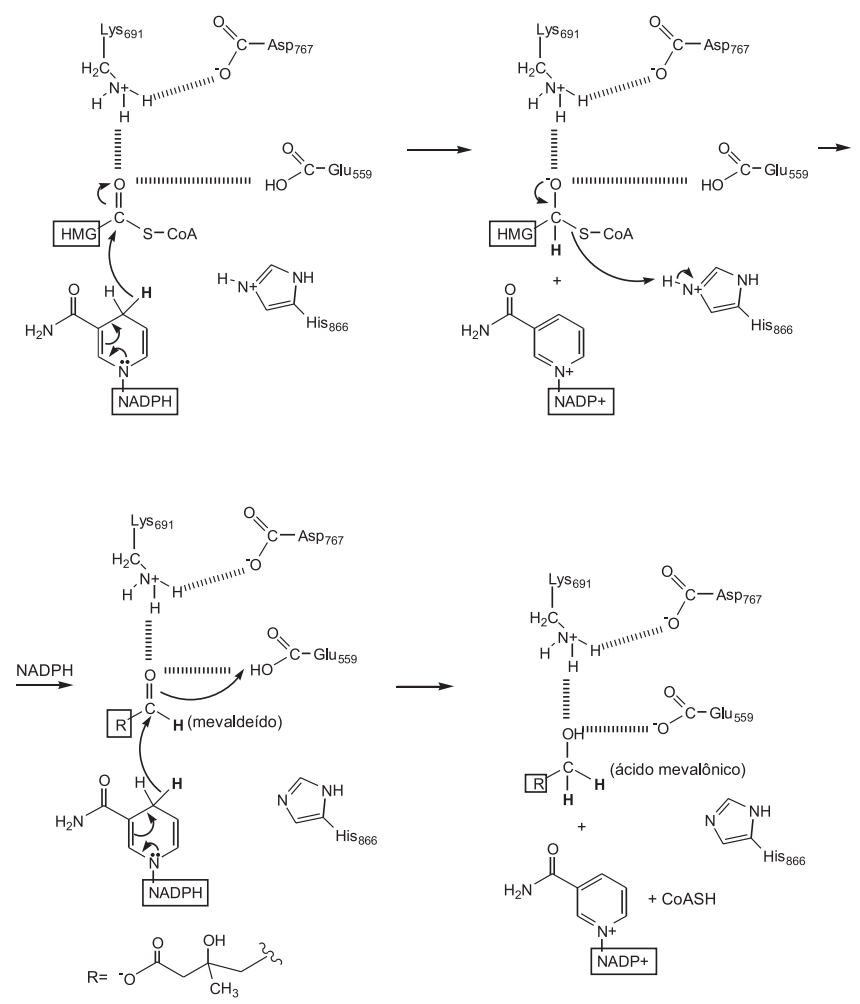

Figura 4. Mecanismo molecular e principais interações do substrato $H M G$ CoA e mevaldeído com aminoácidos do sítio catalítico da enzima HMGR, na presença de duas moléculas de NADPH, envolvidos na formação de ácido mevalônico

Hidroximetilglutaril-CoA redutase (HMGR) apresenta-se na forma de um tetrâmero, no qual dois dos quatro monômeros interagem para formar o sítio catalítico. O grupo hidroximetilglutarílico (HMG) do substrato HMG-CoA posiciona-se na interface destes monômeros, onde ocorrem as reações de redução.

O sítio catalítico de HMGR possui uma região catalítica importante, denominada alça-cis (aminoácidos ligados de forma cis), envolvendo os resíduos 682-694, característicos apenas dos organismos eucariotes. Na região dos aminoácidos C688 a T689, a alça cis está adequadamente posicionada para orientar os resíduos fundamentais que participam das reações de redução (Ser684, Asp690, Lys691 e Lys692). As principais interações do substrato HMG-CoA no sítio catalítico de HMGR estão descritas na Tabela 1.

As investigações realizadas em diversos complexos enzimáticos têm sugerido que existe apenas uma diferença fundamental entre as interações do substrato e as diferentes estatinas. No complexo da enzima com as estatinas parece que ocorre uma mudança conformacional na região flexível C-terminal, que permite a acomodação dos grupos rígidos e hidrofóbicos dos inibidores, aumentando as interações hidrofóbicas com a enzima e contribuindo para a alta potência apresentada pelas estatinas.

\section{RELAÇÃO ESTRUTURA-ATIVIDADE}

De forma geral, os fármacos naturais e sintéticos possuem gru- 
Tabela 1. Principais interações do substrato HMG-CoA no sítio catalítico de HMGR

\begin{tabular}{|c|c|c|c|}
\hline $\begin{array}{l}\text { Grupo funcional } \\
\text { do substrato }\end{array}$ & $\begin{array}{l}\text { Resíduo que realiza } \\
\text { interação }\end{array}$ & Força de interação & Comentários \\
\hline $1-\mathrm{C}=\mathrm{O}$ & Glu559, Lys691* & Ligação de hidrogênio & $\begin{array}{l}\text { Promove a polarização da carbonila } \\
\text { para subseqüente transferência de hidreto }\end{array}$ \\
\hline $3-\mathrm{C}-\mathrm{OH}$ & Asp690, Arg590 & Ligação de hidrogênio & \\
\hline $3-\mathrm{C}-\mathrm{CH}_{3}$ & Leu853 & van der Waals & \\
\hline $5-\mathrm{C}-\mathrm{OH}$ & Lys 735 & Ponte salina & $\begin{array}{l}\text { Região terminal } \mathrm{COO}^{-} \text {, cuja carga é } \\
\text { estabilizada por ambos resíduos }\end{array}$ \\
\hline $5-\mathrm{C}=\mathrm{O}$ & Ser684 & Ligação de hidrogênio & \\
\hline$-\mathrm{SH}(\mathrm{CoA})$ & His 866 & Ligação de hidrogênio & $\begin{array}{l}\text { Posicionado adequadamente próximo ao sítio } \\
\text { para doar hidrogênio ao grupo tiol de CoA }\end{array}$ \\
\hline
\end{tabular}

* O resíduo de Lys691 realiza adicionalmente ligação de hidrogênio com os resíduos Glu559 e Asp767.

pos lipofílicos ligados a uma cadeia lateral comum, representada por unidade de ácido 3,5-di-hidroxivalérico ou $\beta, \delta$-di-hidroxiheptanóico, com substituintes na posição 7, com grande analogia estrutural não só com o substrato natural HMG-CoA da enzima HMG-CoA redutase, mas também com o produto da ação enzimática, ácido mevalônico ${ }^{21}$.

As estatinas são inibidores competitivos da enzima HMGR bastante potentes, com valores de constante de inibição (Ki) na faixa nanomolar (0,1 a 2,3). Por ex., a afinidade de HMG-CoA redutase por mevastatina e lovastatina é, respectivamente, 7140 e 16700 vezes superior que pelo substrato, confirmando a presença de duas regiões de ligações fundamentais no sítio ativo da enzima, uma para ligação do grupo hidroximetilglutaril, no qual o substrato ou a porção de ácido 3,5-di-hidroxivalérico de estatinas se ligam e, uma região adjacente hidrofóbica para complexação do grupo volumoso das estatinas ${ }^{22}$. As estatinas mais potentes devem ser capazes de fornecer grupos carboxilato e hidroxílicos não substituídos, preservando a função ácido 3,5-di-hidroxivalérico (na forma hidroxi-ácido ou lactona). O derivado 5-cetônico representa, no entanto, uma exceção, sua potência, similar ao derivado hidroxílico, deve ser devida à redução do grupo carbonílico in vivo.

Todas as estatinas possuem um grupo hidroximetilglutarílico (HMG) que se liga na mesma região enzimática do grupo HMG do substrato, bloqueando seu acesso ao sítio catalítico. A Figura 5A ilustra as interações moleculares mais relevantes do grupo HMG do substrato no sítio catalítico. Por outro lado, a Figura 5B mostra, comparativamente, as interações do inibidor rosuvastatina complexado no sítio catalítico de HMGR. Outras interações são observadas entre a base adenina e o grupo fosfato do substrato e a molécula de NADPH, mas a Tabela 1 e a Figura 5 se referem apenas às interações relevantes do sítio catalítico.

Pontes de grupo etil ou (E)-etenil fornecem a melhor distância entre o carbono 5-carbinólico e a região lipofílica de decalina das estatinas de primeira geração, enquanto a cadeia lateral de lovastatina, contendo grupo éster 2(S)-metilbutírico $\left[\mathrm{CH}_{3} \mathrm{CH}_{2} \mathrm{CH}\left(\mathrm{CH}_{3}\right) \mathrm{CO}_{2}^{-}\right]$, pode sofrer pequena modificação como a introdução de grupo metílico adicional na posição $\alpha$. Esta variação deu origem à sinvastatina $\left[\mathrm{CH}_{3} \mathrm{CH}_{2} \mathrm{C}\left(\mathrm{CH}_{3}\right)_{2} \mathrm{CO}_{2}^{-}\right]$, cerca de 2,5 vezes mais ativa que lovastatina ${ }^{22}$. Modificações do grupo lipofílico, como substituição de decalina (mevinolina, lovastatina, sinvastatina e pravastatina) por anéis heterocíclicos substituídos, como indol (fluvastatina), piridina (cerivastatina), pirrol (atorvastatina) e pirimidina (rosuvastatina) conduzem a derivados que preservam a atividade hipolipêmica ${ }^{7}$. Os grupos volumosos das estatinas, contendo grupo decalina ou anéis heterocíclicos, adotam diferentes conformações na interação com o sítio de interação que conduzem a interações hidrofóbicas.

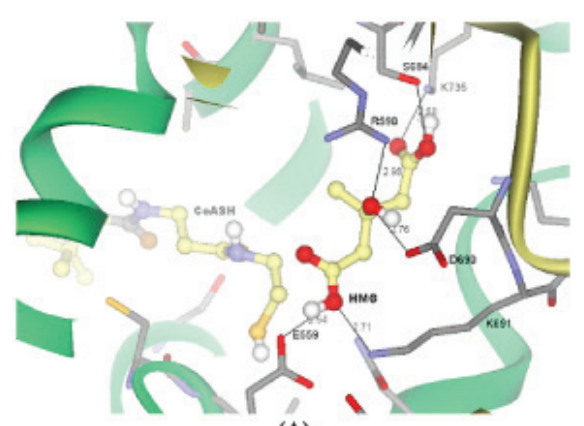

(A)

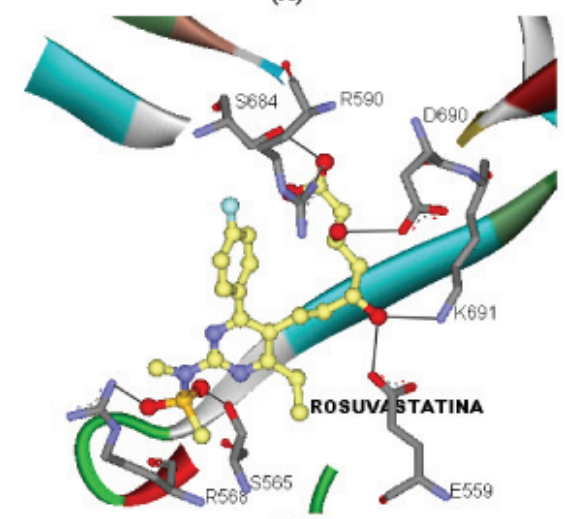

(B)

Figura 5. Interações do substrato (5A), extraído do PDB código ID 1DQA, e inibidor rosuvastatina (5B), código ID $1 H W L$, complexadas no sítio catalítico da enzima HMG-CoA redutase. A Figura 5 A ilustra as interações mais importantes da unidade de ácido 3-hidróxi-metilglutarílico do substrato, após clivagem e liberação de CoASH. A Figura 5B mostra, comparativamente, as interações do inibidor rosuvastatina, semelhantes ao substrato

As estatinas sintéticas possuem um grupo 4-fluorofenil que participa de interações adicionais através do anel aromático e do átomo de flúor com o grupo guanidínico do resíduo Arg590. Por outro lado, a cadeia lateral de ácido butírico das estatinas, contendo grupo decalina, como pravastatina, ocupa regiões semelhantes no sítio catalítico. Além destas interações, atorvastatina e rosuvastatina contém uma cadeia lateral polar com grupo carbonila ou sulfonamida, respectivamente, que realiza interação por ligação de hidrogênio com o resíduo de Ser565. Somente rosuvastatina realiza interação polar com o resíduo de Arg568, pela interação com grupo sulfonamido ${ }^{23}$. As interações moleculares da enzima complexada com diferentes estatinas ou substrato estão disponíveis no PDB ("Protein Data Bank").

Pravastatina é usada na forma de hidroxi-ácido livre, enquanto 
mevastatina, lovastatina e sinvastatina são usadas como pró-farmacos na forma de lactonas, que serão enzimaticamente convertidas pela ação de esterases aos correspondentes hidroxi-ácidos (ácido 3,5-dihidroxivalérico), ativos para inibição de HMG-CoA redutase. Pravastatina é o metabólito de mevastatina, enquanto sinvastatina é análogo de lovastatina com grupo metílico adicional na cadeia lateral. In vivo, a forma lactônica de pró-fármacos de estatinas está em equilíbrio reversível com a forma hidroxi-ácida ativa, capaz de mimetizar estruturalmente o substrato natural HMG-CoA.

A molécula de mevinolina, assim como as estatinas clássicas, possui sete centros assimétricos, mas estudos de Relação Estrutura Atividade (REA) têm mostrado que pelo menos cinco destes centros quirais, presentes na porção hexa-hidronaftalênica e cadeia lateral (posição 8), são desnecessários para inibição efetiva de HMGCoA redutase. Por outro lado, a configuração do carbono $\delta$ dos derivados lactônicos parece importante, pois o epímero de lovastatina não apresenta qualquer atividade hipolipêmica ${ }^{7}$. Atorvastatina e rosuvastatina são exemplos de estatinas que preservam apenas dois centros assimétricos da molécula de mevastatina original e, mesmo assim, são potentes inibidores enzimáticos².

Os pró-fármacos lovastatina e sinvastatina são menos solúveis em água, enquanto pravastatina, mais hidrossolúvel, possui baixa penetração em células não hepáticas, maior seletividade para inibição de HMG-CoA redutase hepática e ínicio de ação mais rápida, atingindo picos de concentração em apenas $1 \mathrm{~h}$.

\section{NOVAS TENDÊNCIAS TERAPÊUTICAS - EFEITOS PLEIOTRÓPICOS}

As estatinas exercem muitos efeitos independentes da diminuição do colesterol sérico, conhecidos como pleiotrópicos ${ }^{6}$. Estes efeitos incluem ação reguladora na função endotelial, aumento da estabilidade de placas ateroscleróticas, diminuição do estresse oxidativo e inflamação, e diminuição da resposta trombogênica. Além destes, as estatinas possuem efeitos extra-hepáticos benéficos no sistema imune, sistema nervoso central e ósseo. Muitos destes efeitos pleiotrópicos são mediados pela inibição de isoprenóides, os quais servem como ligantes lipídicos para moléculas envolvidas em processos de sinalização celular.

\section{Função endotelial}

Uma importante característica da disfunção endotelial é a síntese ineficaz, liberação e atividade do óxido nítrico derivado de endotélio (NO). O óxido nítrico endotelial parece inibir vários componentes de processos aterogênicos, por ex., mediando relaxamento vascular e inibição de agregação plaquetária ${ }^{24}$. Estudos prévios afirmaram que as estatinas poderiam restabelecer a função endotelial, em parte, pela diminuição dos níveis de colesterol sérico. No entanto, foi verificado que o restabelecimento da função endotelial ocorreu anteriormente à diminuição do colesterol, sugerindo que efeitos adicionais à redução do colesterol estão envolvidos neste processo, principalmente o aumento de óxido nítrico endotelial por estimulação e sub-regulação da enzima óxido nítrico sintase $(\mathrm{eNOS})^{25}$.

As estatinas também têm sido vinculadas à capacidade de aumento do número de células endoteliais progenitoras circulantes (EPCs), além de induzirem a angiogênese através da proliferação, migração e sobrevida das células EPCs circulantes ${ }^{26}$. Em pacientes com doença arterial coronária estável, a administração de estatinas por quatro semanas aumentou o número de células circulantes EPCs, promovendo, conseqüentemente, melhora na capacidade circulatória destes pacientes.

\section{Inflamação vascular}

Estudos recentes sugerem que as estatinas possuem propriedades antiinflamatórias devido à capacidade de reduzirem o número de células inflamatórias em placas ateroscleróticas ${ }^{27}$. Os mecanismos envolvidos estão relacionados à inibição da adesão de moléculas, tais como moléculas de adesão intercelular (ICAM-1), as quais estão ligadas ao recrutamento de células inflamatórias. Adicionalmente, as estatinas atenuam a expressão de P-selectinas e a adesão de leucócitos pelo aumento da produção de óxido nítrico endotelial. Este efeito independente de colesterol das estatinas não foi observado em ratos deficientes em eNOS, sugerindo que esta enzima foi mediadora nos efeitos vasculares protetores das estatinas.

\section{Demência}

Dados epidemiológicos recentes têm mostrado que as estatinas podem exercer efeitos protetores contra a doença de Alzheimer e outros tipos de demência. A doença de Alzheimer está relacionada aos efeitos da proteína $\beta$-amilóide, a qual pode se acumular no cérebro, causando neurotoxicidade e neurodegeneração. Estudos experimentais e clínicos sugerem que há uma relação patofisiológica entre proteína $\beta$-amilóide e níveis séricos de colesterol, sendo que níveis aumentados desta proteína e da apolipoproteína E (ApoE) são fatores de risco para a doença de Alzheimer ${ }^{28}$. Desta forma, independentemente das estatinas parecerem induzir alterações na distribuição de colesterol celular no cérebro, tais efeitos exercidos pelas estatinas podem ser mediados via $\mathrm{NO}$ ou $\mathrm{ApoE}^{29}$.

Segundo pesquisas realizadas, a prevalência de doença de Alzheimer em pacientes tratados com estatinas é $60 \%$ mais baixa em comparação a pacientes que fazem uso de outras medicações utilizadas no tratamento de doenças cardiovasculares. Assim sendo, evidências recentes têm estimulado a investigação de estatinas como potenciais novos agentes antiinflamatórios e protetores vasculares para o tratamento de outras doenças cerebrais, tais como esclerose múltipla e depressão.

\section{Neoplasias}

As estatinas têm sido relacionadas às propriedades de inibição de proliferação e indução de apoptose em uma grande variedade de células tumorais, sendo desencadeados efeitos antitumorais em modelos animais contra melanoma, carcinoma mamário, adenocarcinoma pancreático, fibrosarcoma, glioma, neuroblastoma e linfoma, resultando em retardamento de crescimento tumoral e inibição de processos metastáticos. Em estudos pré-clínicos, as estatinas têm demonstrado a capacidade de potencializar os efeitos antitumorais de algumas citocinas e quimioterápicos.

Lovastatina é a estatina mais estudada no que se refere a potenciais efeitos antitumorais, tendo sido capaz de exercer atividade antiproliferativa contra uma variedade de tumores, incluindo carcinoma de seio, próstata, estômago, pulmão, pâncreas, bexiga, adenocarcinoma de cólon, neuroblastoma, melanoma e células de leucemia mielóide aguda ${ }^{30,31}$. Efeitos citostáticos contra células tumorais também têm sido observados com o uso de pravastatina, sinvastatina, fluvastatina e cerivastatina ${ }^{30}$. Lovastatina e outras estatinas também inibiram o crescimento de várias células normais e linhagens de células não transformadas, incluindo células de músculo liso, mioblastos de músculo estriado, células endoteliais, fibroblastos, células do epitélio alveolar e linfócitos B, mas células normais parecem ser menos sensitivas aos efeitos antiproliferativos das estatinas se comparadas às células tumorais ${ }^{32}$.

Com relação a efeitos antimetastáticos, lovastatina tem inibido 
etapas essenciais de processos metastáticos, nomeados ligação, motilidade e invasão, sendo que fluvastatina e cerivastatina também suprimiram a invasão de células tumorais humanas ${ }^{33}$. As estatinas foram capazes de inibir metástase de células de melanoma e células de carcinoma mamário, bem como metástase de células de adenocarcinoma de cólon, em ratos.

\section{Associação de estatinas a outros tratamentos}

As estatinas têm demonstrado a capacidade de potencializar a atividade antitumoral de algumas citocinas e agentes quimioterápicos. Dentre estes agentes quimioterápicos estão cisplatina, 5-fluorouracil, paclitaxel e doxorubicina. Lovastatina foi capaz de aumentar a apoptose induzida por cisplatina em quatro linhas de células de adenocarcinoma de cólon e, sinergisticamente, potencializar a atividade de paclitaxel contra linhagens de células humanas de leucemia, in vitro, e de cisplatina contra melanoma de murina, tanto in vitro quanto in vivo ${ }^{34}$. O mecanismo preciso envolvido nesta interação sinérgica entre lovastatina e cisplatina em células tumorais ainda não foi estabelecido. No entanto, considerando que lovastatina detém uma variedade de células na fase G1 do ciclo celular e estas células detidas parecem mostrar sensibilidade aumentada para cisplatina, não pode ser excluída a hipótese de que lovastatina torne as células tumorais mais susceptíveis à ação de cisplatina ${ }^{35}$. Já as interações entre lovastatina e 5-fluorouracil ou doxorubicina parecem ser particularmente interessantes. Lovastatina tem sido capaz de fortalecer a atividade pró-apoptótica de 5-fluorouracil em linhagens de células de carcinoma de cólon humano e potencializar os efeitos antitumorais de doxorubicina contra melanoma de murina, carcinoma de cólon e carcinoma de pulmão, tanto in vitro quanto in $v i v o^{36}$. Lovastatina não somente aumentou a atividade terapêutica de doxorubicina nestes modelos de tumores de murina, mas reduziu significativamente sua cardiotoxicidade ${ }^{36}$.

As estatinas têm sido capazes de, efetivamente e até mesmo sinergisticamente, potencializar a atividade antitumoral de determinadas citocinas e quimioterápicos em uma grande variedade de modelos tumorais experimentais. Também pode-se supor que pacientes com câncer que não tenham sido tratados com estatinas previamente ao desenvolvimento do tumor poderiam se beneficiar destes fármacos incluídos como adjuvantes à terapia convencional, aumentando, portanto, sua efetividade e adicionalmente, diminuindo os riscos de doenças cardiovasculares.

\section{REFERÊNCIAS}

1. http:/www.who.ch., acessada em Abril 2005.

2. Farnier, M.; Davignon, J.; Am. J. Cardiol. 1998, 82, 3.

3. Sierra, M. Em Burger's Medicinal Chemistry and Drug Discovery; Abraham, D. J., ed.; $6^{\text {th }}$ ed.; Wiley-Interscience: New Jersey, 2003, vol. 3, p. 339.

4. http://portal.saude.gov.br/saude/aplicacoes/anuario2001/index.cfm, acessada em Dezembro 2003.
5. Witztum, J. L. Em As Bases Farmacológicas da Terapêutica; Hardman, J. G.; Limbird, L. E.; Molinoff, P. B.; Ruddon, R. W.; Goodman Gilman, A., eds., $9^{\text {th }}$ ed.; McGraw Hill: Rio de Janeiro, 1997, p. 643.

6. Liao, J. K.; Laufs, U.; Annu. Rev. Pharmacol. Toxicol. 2005, 45, 89.

7. Wierzbicki, A. S.; Mikhailidis, D. P.; Wray, R.; Schachter, M.; Cramb, R.; Simpson, W. G.; Byrne, C. B.; Curr. Med. Res. Opin. 2003, 19, 155.

8. Bakri, R.; Wang, J.; Wierzbicki, A. S.; Goldsmith, D.; Int. J. Cardiol. 2003, 91, 107.

9. Jakobisiak, M.; Golab, J.; Int. J. Oncol. 2003, 23, 1055.

10. Staal, A.; Frith, J. C.; French, M. H.; Swartz, J.; Gungor, T.; Harrity, T. W.; Tamasi, J.; Rogers, M. J.; Feyen, J. H.; J. Bone Min. Res. 2003, 18, 88.

11. Kalinowski, L.; Dobrucki, L. W.; Brovkovych, V.; Malinski, T.; Circulation 2002, 105, 933.

12. Mukai, Y.; Shimokawa, H.; Matoba, T.; Hiroki, J.; Kunihiro, I.; Fujiki, T.; Takeshita, A.; J. Cardiol. Pharmacol. 2003, 42, 118.

13. Nishikimi, T.; Tadokoro, K.; Wang, X.; Mori, Y.; Asakawa, H.; Akimoto, K.; Yoshihara, F.; Horio, T.; Minamino, N.; Matsuoka, H.; Eur. J. Pharmacol. 2002, 453, 175.

14. Vincent, L.; Soria, C.; Mirshahi, F.; Opolon, P.; Mishal, Z.; Vannier, J. P.; Soria, J.; Hong, L.; Arterioscler. Thromb. Vasc. Biol. 2002, 22, 623.

15. Van Harmelen V.; Skurk, T.; Rohrig, K.; Haumer, H.; Horm. Metab. Res. 2003, 35, 466.

16. Ver "Editorial", The statin wars: why AstraZeneca must retreat, The Lancet 2003, 362 (9393), 1341

17. Kajinami, K.; Takekoshi, N.; Saito, Y; Cardiovasc. Drug Rev. 2003, 21, 199.

18. Mukhtar, R. Y. A.; Reid, J.; Reckless, J. P. D.; Int. J. Clin. Pract. 2005, 59, 239.

19. Wermuth, C. G. Em The Practise of Medicinal Chemistry; Wermuth, C. G., ed.; Academic Press: San Diego, 1996, p. 243 e 304.

20. Mayes, P. A. Em Harper's Biochemistry Murray, R. K.; Granner, D. K.; Mayes, P. A.; Rodwell, V. W., eds.; 24ª ed., Appleton \& Lange: Stanford, 1996, p. 271.

21. Lea, A. P.; McTavish, D.; Drugs 1997, 53, 828

22. Leitersdorf, E.; Br. J. Clin. Pract. 1994, Suppl. 77, 24.

23. Instvan, E. S.; Deisenhofer, J.; Science 2001, 292, 1160.

24. Radomski, M. W.; Rees, D. D.; Dutra, A.; Moncada, S.; Br. J. Pharmacol. 1992, 107, 745.

25. Kureishi, Y.; Luo, Z.; Shiojima, I.; Bialik, A.; Fulton, D.; Nat. Med. 2000, 6, 1004.

26. Dimmeler, S.; Aicher, A.; Vasa, M.; Mildnerrihm, C.; Aldler, K.; J. Clin. Invest. 2001, 108, 391.

27. Vaughan, C. J.; Gotto, A. M. Jr.; Basson, C. T.; J. Am. Coll. Cardiol. 2000, 35,1 .

28. Corder, E. H.; Saunders, A. M.; Strittmatter, W. J.; Schmechel, D. E.; Gaskell, P. C.; Science 1993, 261, 921.

29. Kirsh, C.; Eckert, G. P.; Koudinov, A. R.; Muller, W. E.; Pharmacopsychiatry 2003, 36 (Suppl. 2), S113.

30. Newman, A.; Clutterbuck, R. D.; Powles, R. I.; Catovsky, D.; Millar, J. L.; Leuk. Lymphoma 1997, 24, 533.

31. Newman, A.; Clutterbuck, R. D.; Powles, R. I.; Millar, J. L.; Leukemia 1994, 8, 274.

32. Murakami, M.; Goto, T.; Saito, Y.; Goto, S.; Kochi, M.; Ushio, Y.; Int. J. Oncol. 2001, 19, 525.

33. Denoyelle, C.; Vasse, M.; Korner, M.; Mishal, Z.; Ganne, F.; Vannier, J.-P.; Soria, J.; Soria, C.; Carcinogenesis 2001, 22, 1139.

34. Feleszko, W.; Zagozdzon, R.; Golab, J.; Jakobisiak, M.; Eur. J. Cancer 1998, 34, 406.

35. Keyornarsi, K.; Sandoval, L.; Band, V.; Pardee, A. B.; Cancer Res. 1991, 51,3602 .

36. Feleszko, W.; Mlynarezuk, I.; Balkowile-Iskra, E. Z.; Clin. Cancer Res. 2000, 6, 2044. 